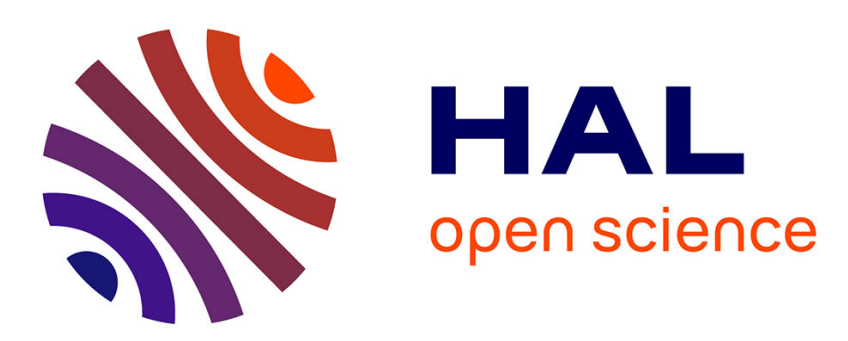

\title{
La pratique des mutilations génitales féminines à Djibouti : une " affaire de femmes " entre les mains des hommes
}

Severine Carillon, Véronique Petit

\section{- To cite this version:}

Severine Carillon, Véronique Petit. La pratique des mutilations génitales féminines à Djibouti : une " affaire de femmes " entre les mains des hommes. Autrepart - Revue de sciences sociales au Sud, 2009, 52 (4), pp.13-29. 10.3917/autr.052.0013 . halshs-02113380

\section{HAL Id: halshs-02113380 \\ https://shs.hal.science/halshs-02113380}

Submitted on 19 Aug 2021

HAL is a multi-disciplinary open access archive for the deposit and dissemination of scientific research documents, whether they are published or not. The documents may come from teaching and research institutions in France or abroad, or from public or private research centers.
L'archive ouverte pluridisciplinaire HAL, est destinée au dépôt et à la diffusion de documents scientifiques de niveau recherche, publiés ou non, émanant des établissements d'enseignement et de recherche français ou étrangers, des laboratoires publics ou privés. 


\title{
Décider de la pratique des mutilations génitales féminines à Djibouti : « une affaire de femmes »?
}

\author{
Séverine CARILLON et Véronique PETIT
}

Les Mutilations Génitales Féminines (MGF) sont encore largement pratiquées sur le continent africain puisqu'elles touchent environ 75 millions de femmes et enfants dans 26 pays d'Afrique. La République de Djibouti détient la prévalence la plus élevée d'Afrique orientale : en 2002, près de $98 \%$ des femmes âgées de 15 à 49 ans déclaraient avoir subi une $\mathrm{MGF}^{1}$. La nature de l'opération varie d'un pays à l'autre et d'un groupe ethnique à un autre. A Djibouti, trois types de mutilations sont repérables sur l'ensemble de la population : l'infibulation (type 1) forme la plus sévère, l'excision (type 2) et la sunna (type 3) forme la plus légère. Quelles qu'elles soient, ces pratiques constituent une atteinte à l'intégrité physique et morale des femmes et contribuent à la surmortalité maternelle et infantile. En conséquence, elles sont contraires à tous les textes et déclarations universelles protégeant l'individu². Elles sont, à Djibouti, interdites et condamnables par l'Etat depuis 1995.

La lutte contre les mutilations génitales féminines y a été amorcée au début des années 1980. Une démarche politique et sociale a, depuis, amené les autorités politiques et certains acteurs de la société civile à mettre progressivement la question des MGF au cœur des débats qui accompagnent le changement social à Djibouti. Cette mobilisation institutionnelle nationale, soutenue et renforcée par les partenaires internationaux en développement, a conduit à intégrer des questions relatives à la pratique et aux attitudes relatives aux MGF dans des grandes enquêtes démographiques.

Ainsi, les résultats de l'Enquête démographique et sanitaire PAPFAM ${ }^{3}$ réalisée en $2002^{4}$, font état de la gravité de la situation à Djibouti. Parmi les femmes enquêtées, âgées de 15 à 49 ans, ayant subi une MGF, $75,5 \%$ ont subi une infibulation. La prévalence de l'infibulation à Djibouti est inférieure à celle des districts : 74,7\% contre $81,3 \%$. De même, on constate une surreprésentation des mutilations les plus légères (sunna) à Djibouti par rapport aux districts $(10,6 \%$ contre $1,9 \%)$. On observe par ailleurs, sur l'ensemble du pays, une transformation dans la répartition des types de mutilations avec une diminution progressive de l'infibulation pour les femmes les plus jeunes et une augmentation corollaire de la sunna.

L'Enquête pilote sur la médecine scolaire préventive à Djibouti ${ }^{5}$, réalisée en 2006 auprès des petites filles âgées de cinq à dix ans dans dix écoles primaires publiques, montre quant à elle, une baisse sensible du taux de prévalence des MGF et du pourcentage d'infibulation. On peut

\footnotetext{
${ }^{1}$ DISED (Direction des Statistiques et des études démographiques), Enquête démographique et sanitaire, Djibouti, 2002.

${ }^{2}$ La Déclaration Universelle des Droits de l'Homme; La Convention relative aux droits de l'enfant ; La Convention sur l'élimination de toutes les formes de violence à l'égard des femmes ; La Déclaration sur l'élimination de la violence à l'égard des femmes.

${ }^{3}$ Programme Arabe sur la Santé de la Famille

${ }^{4}$ DISED (Direction des Statistiques et des études démographiques), Enquête démographique et sanitaire, Djibouti, 2002.

${ }^{5} \mathrm{O}$. BELBEOCH,

2006. La Médecine scolaire préventive à Djibouti. Enquête pilote 2005-2006. Atelier du 30 septembre 2006. Paris, POPINTER, UNFPA, 16p.

- 2006. La médecine scolaire à Djibouti. Enquête pilote 2005-2006. Paris, POPINTER, UNFPA. 24p.
} 
en effet estimer à presque une sur cinq la proportion de filles, à 13 ans, sans aucune mutilation dans le district de Djibouti.

La question de ne pas exciser les fillettes est désormais posée dans les familles djiboutiennes. Comment et par qui la réponse à cette question est-elle élaborée ?

En s'inscrivant dans une approche résolument anthropologique, nous chercherons à reconstruire et comprendre le processus de décision élaborée au sein de la cellule familiale qui conduit à l'acceptation ou au refus de la pratique d'une MGF sur les fillettes. Comment la décision d'exciser ou non les fillettes est-elle prise ? Quels sont les acteurs qui participent à cette décision? Qui l'influence? Quels sont les facteurs qui la déterminent ? Quels sont les arguments mobilisés? L'enjeu de la question est de comprendre comment les hommes et les femmes passent d'un environnement favorable aux MGF à un environnement opposé à ces pratiques.

Ce questionnement sera abordé à partir d'une enquête qualitative réalisée à Djibouti-ville. Vingt-cinq entretiens semi-directifs ont été conduits en novembre et décembre 2006 auprès d'hommes et de femmes âgés de 18 à plus de 60 ans, ayant ou non imposé la pratique d'une MGF à leur(s) fille(s). La moitié des entretiens a été réalisée en langue locale (le Somali) avec une interprète. La sélection des individus répond à des critères de diversification tant ethnique que sociale et économique.

Cette question récurrente et inévitable - imposer une MGF à sa fille - se pose à des individus, à des familles qui vivent et agissent dans un cadre particulier. La République de Djibouti est un Etat récent (1977). C'est une société multiculturelle en raison de son histoire et de la composition de sa population. Il s'agit d'une République islamique, donc d'une société encore profondément marquée par la religion. La totalité, ou presque, de la population djiboutienne se déclare musulmane. C'est une société ouverte qui, étant donné l'histoire de son peuplement, par l'épisode colonial, et par sa position géographique, est soumise à des influences aussi diverses et contradictoires que celles de l'Occident, de la Péninsule arabique et de l'Afrique Noire. En conséquence, elle se construit et évolue en référence à des modèles sociaux, culturels et économiques différents. Enfin, c'est une société en pleine évolution : la modernisation du pays, de son économie, la construction de l'Etat djiboutien ne peuvent pas être sans conséquence sur l'organisation sociale de la société. Ces caractéristiques expliquent en partie les tensions autour du processus de décision relatif à la pratique ou à l'abandon des MGF. L'analyse de ce processus décisionnel passe donc, en amont, par une analyse du contexte initial.

\section{1- Le contexte de la lutte contre les MGF}

\section{1-1: Les enjeux sociaux de l'abandon des MGF}

La promulgation de la loi interdisant les MGF à Djibouti, en 1995, introduit, au moins en théorie, un changement de comportement : une pratique jusque là autorisée et largement répandue devient interdite et condamnable. Ce qui était licite et entretenu sur des générations est brutalement banni. La norme n'est plus la fillette mutilée mais la fillette non-mutilée. Un nouveau modèle de référence ${ }^{6}$ entre donc en vigueur dans la société djiboutienne. Prônant des valeurs occidentalisées, il repose sur la reconnaissance des MGF comme une violation du

\footnotetext{
${ }^{6}$ Le modèle de référence est un ensemble de significations, de règles, de normes auxquelles les individus se réfèrent pour orienter leurs actions.
} 
droit à l'intégrité physique, à disposer de son propre corps, et sur le refus de la souffrance. Les autorités politiques tentent de substituer ce modèle à un autre, plus ancien. Ce dernier se caractérise par la pratique systématique des MGF sur les fillettes et particulièrement la pratique de l'infibulation. Il repose sur une tradition forte et sur un ensemble de croyances. Le passage d'un modèle de référence à un autre génère des transformations considérables dans la société djiboutienne.

\section{- Le bouleversement de l'organisation sociale djiboutienne}

La perpétuation des MGF, d'une part, assure un lien de continuité entre les générations : les jeunes adoptent les comportements que leurs aînées leur ont implicitement inculqués, toutes ont été victimes d'une mutilation, toutes ont connu et connaissent les souffrances qui en résultent... Autant de points communs et de souffrances partagées qui soudent le groupe et rappellent chaque fois la souffrance des aînées mais aussi leur courage qui font l'honneur de la famille.

D'autre part, la perpétuation des MGF renvoie à des pratiques coutumières qui ont jusque-là parfaitement rempli leur rôle de contrôle et d'intégration des femmes dans les organisations sociales traditionnelles. En effet, en subissant une MGF, la femme devient conforme aux autres membres de sa tribu et est intégrée au groupe. Cette pratique contribue ainsi à la cohésion du groupe qui l'exerce et rappelle leurs origines communes.

En outre, l'intervention sur le sexe féminin apparaît comme un moyen de préservation de la chasteté des femmes par inhibition du désir sexuel : chez la femme mariée, elle réduirait les risques de céder aux tentations adultérines; chez la jeune fille, elle est censée éviter toute velléité de rapport sexuel prématurée et illicite, c'est-à-dire hors mariage, et toute grossesse prématurée, c'est-à-dire hors mariage également. La pratique des MGF favorise ainsi le contrôle de la sexualité des femmes et, a fortiori, permet aux hommes de s'assurer de la fidélité de leurs femmes et de l'origine de leur progéniture. En définitive, elle entretient la domination masculine.

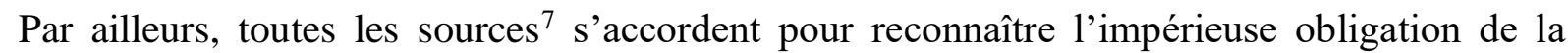
virginité comme motivation principale de la pratique des MGF. Celle-ci témoigne de l'intérêt porté à la virginité des filles jusqu'au mariage. En effet, dans la société djiboutienne, la virginité des jeunes filles est un élément incontournable qui rend possible le mariage. Par conséquent, subir une MGF, c'est accéder au mariage, obtenir le droit de procréer, de devenir féconde, et ainsi entrer dans le cycle de l'échange matrimonial et enfin, accéder à la reconnaissance sociale. Inversement, l'abandon de la pratique équivaut pour une jeune fille à la condamnation au célibat, ce qui, dans une société ou le mariage est d'une part la seule porte d'entrée au statut d'adulte (que ce soit pour un homme ou une femme) et d'autre part, une obligation pour prendre sa place dans la société et y être reconnu, signifie une marginalisation sociale. Le célibataire n'est effectivement pas en position de remplir son devoir - contribuer à la reproduction du groupe - vis-à-vis de sa communauté d'appartenance. Ainsi, l'abandon des MGF introduit un bouleversement du système matrimonial.

\section{- La remise en cause d'un système idéologique}

Le changement de pratiques qu'est censé exiger la loi interdisant les MGF, implique de remettre en cause des comportements qui, jusque-là, allaient de soi. En effet, la pratique des MGF s'inscrivait dans l'ordre immémorial des choses, c'était l'essence de l'ordre social, la normalité. Conçue par tous comme une évidence, nul ne la remettait en cause. «Cela s'est

\footnotetext{
${ }^{7}$ Voir M. Erlich, La femme blessée : essai sur les mutilations sexuelles, Paris, l'Harmattan, 1986.
} 
toujours fait, donc cela doit continuer. Vieil immobilisme qui justifie tout et élimine d'entrée toute réflexion novatrice » (Prolongeau, 2006 : 154) Cette évidence, largement intériorisée par les femmes et constitutive de leur identité, est donc brutalement bouleversée.

D'une manière plus générale, c'est une rupture avec l'ensemble des systèmes explicatifs développés depuis des générations pour justifier de la pratique des MGF qui s'opère.

La perpétuation des MGF s'appuie sur des discours et des croyances - avant tout religieusesun réseau complexe de significations, qui justifient ces interventions. Ainsi, des arguments esthétique et hygiénique selon lesquels les organes génitaux féminins non touchés sont laids, sales et encombrants ont été mentionnés par les personnes enquêtées. Le sexe féminin supposé laid doit être enjolivé par sa transformation en partie lisse et sans protubérance. Cet argument repose sur une croyance ancienne que le sexe féminin, ouvert et fait de replis, serait forcément réceptif à la saleté et aux microbes. La laideur et la saleté se confondant, les tenants de l'excision soutiennent qu'elle est une protection prophylactique contre les microbes et les maladies vénériennes.

En outre, associée à l'hygiène, au devoir moral ou simplement perçue comme «bonne pratique », les MGF sont valorisées. Elles auraient des effets bénéfiques sur les fillettes et les femmes et seraient réalisées dans l'intérêt de tous : pour la victime, puisqu'elle serait en quelque sorte purifiée, pour les initiateurs de l'opération qui accompliraient une mission multiple et salvatrice (rendre la fillette conforme, en bonne santé, et belle selon les canons d'une esthétique définie sexuellement), enfin aussi les hommes qui épouseraient des femmes purifiées, conformes aux souhaits de la société et de la religion.

Ces représentations sont renforcées par des perceptions négatives des fillettes non mutilées. L'opprobre publique, la honte et la stigmatisation s'abattent effectivement sur ces fillettes. Selon les individus interrogés, ne pas se conformer à la pratique pourrait entraîner le blâme, l'exclusion, une perte de statut et de protection. Le risque d'une telle mort sociale a largement été souligné par les personnes rencontrées et est réellement craint. En revanche, le risque d'une mort physique consécutive à d'éventuelles complications n'a pas même été évoqué.

Enfin, l'éradication des MGF implique un changement d'attitudes et d'opinions des populations par rapport à leur conception de la santé et de la mort d'une part, du statut et du droit des femmes et des enfants d'autre part. En effet, le passage d'un modèle à l'autre opère l'un des glissements entre société traditionnelle au sein de laquelle la mort humaine est acceptée tant qu'elle a lieu dans le cadre social et le respect des valeurs (courage, honneur) et la société moderne qui, au contraire, valorise la survie à n'importe quel prix, social ou matériel, et l'expression des affects. Ce modèle est également empreint d'une idéologie occidentale en vogue pour laquelle la moindre souffrance doit être exprimée, prise en charge psychologiquement et socialement, soulagée médicalement. La condamnation de l'excision repose certes sur l'atteinte à l'intégrité du corps de la fille, mais certainement aussi sur le scandale provoqué par une souffrance infligée à la fillette par le maintien de coutumes surannées ou ne se justifiant plus.

Abandonner les MGF, c'est également reconnaître un droit aux enfants en tant que personne, en tant que sujet individualisé à qui on doit le respect de l'intégrité physique, alors que traditionnellement les seules personnes qui peuvent faire valoir des droits sont les hommes adultes. Ce problème de fond rejoint la difficulté qu'ont les femmes dans les procédures de divorce à faire appliquer les décisions de justice (garde des enfants, versement de la pension) qui leur sont favorables, car c'est une remise en question de l'ordre social traditionnel qui n'est pas discutable et négociable par des individus de statut « inférieur » (femmes et enfants).

Si l'éradication des MGF apparaît nécessaire aux yeux de la communauté internationale et des autorités djiboutiennes, elle est cependant lourde de conséquences puisqu'elle implique une 
révision et une remise en cause d'un système idéologique et d'une culture fortement dépendants de l'organisation sociale traditionnelle. Elle génère un changement de fond quelque peu déstabilisateur pour la société djiboutienne. Comment donc ce passage d'un modèle de référence à un autre s'opère-t-il à Djibouti ?

\section{1-2: Un consensus apparent}

\section{- Les MGF à Djibouti : l'émergence d'une question de société ?}

La lutte contre les MGF à Djibouti commence à l'initiative de l'UNFD $^{8}$ par le lancement de campagnes d'informations visant à inciter à la pratique des formes plus légères de mutilations. En 1984, l'UNFD met en place un Comité National de Lutte Contre les Pratiques Traditionnelles Néfastes (CLNPTN), organe chargé de mener les actions concernant les MGF. Une campagne de sensibilisation destinée aux femmes, mais aussi aux leaders politiques et religieux est engagée. Elle vise à montrer les effets néfastes et l'absence de fondement religieux des MGF.

Le début des années 1990 est marqué par quelques avancées notables sur le plan législatif. Le gouvernement djiboutien signe la Convention relative aux droits de l'enfant $^{9}$ ainsi que la Convention sur l'élimination de toutes formes de discrimination envers les femmes ${ }^{10}$. C'est en 1995 que l'Assemblée Nationale adopte l'article 333 du Code Pénal interdisant toutes les formes de violences. La mise en place de ce cadre juridique marque le passage vers la structuration de la lutte contre les MGF.

En 1997, les MGF sont déclarées constituer une priorité de santé publique et dès 1999, le Ministère de la santé lance des actions de recherche ${ }^{11}$. En 2005, le gouvernement djiboutien organise la Conférence sous-régionale sur l'élimination des MGF. En 2006 est formulée la «Stratégie Nationale pour l'Abandon de l'Excision et de l'Infibulation » (SNAEI). Cette stratégie nationale s'appuie sur une démarche qui privilégie la recherche, la formation, la sensibilisation et l'appui institutionnel. Dans ce cadre, un plan d'action national quinquennal est mis en place.

L'ensemble de ces prises de décisions politiques montre que les MGF constituent fin 2006, date de l'enquête, à Djibouti, un problème national du point de vue de certains acteurs et qu'elles sont mises sur le devant de la scène publique. Pourtant, au-delà de cet apparent consensus sur l'éradication de ces pratiques, l'observation de la réalité laisse entrevoir un contexte bien plus complexe puisque pourvu de contradictions, parmi lesquelles la promulgation d'une loi inappliquée et inapplicable.

\section{- L'interdiction des MGF régie par une loi non applicable}

L'article 333 du Code Pénal djiboutien interdit toutes les formes de violences. Selon cet article, «la violence imposée par les mutilations génitales est passible de cinq ans d'emprisonnement et d'une amende d'un million de francs Djiboutiens ». Cette loi émane

\footnotetext{
${ }^{8}$ Union Nationale des Femmes Djiboutiennes.

${ }^{9}$ La Convention internationale sur les droits de l'enfant est entrée en vigueur le 6 septembre 1990. Elle mentionne dans son article 24 que: "Les Etats parties reconnaissent le droit de l'enfant de jouir du meilleur état de santé possible et prennent toutes les mesures efficaces appropriées en vue d'abolir les pratiques traditionnelles préjudiciables à la santé des enfants ". Cette convention a été ratifiée par le gouvernement djiboutien en 1990.

${ }^{10}$ La Convention relative à l'élimination de toutes les formes de discrimination à l'égard des femmes est entrée en vigueur le 13 janvier 1984. Elle stipule dans son article 2 que : «Les Etats parties s'engagent à prendre toutes les mesures appropriées, y compris les dispositions législatives, pour modifier ou abroger toute loi, disposition réglementaire, coutume ou pratique qui constitue une discrimination à l'égard des femmes ".

${ }^{11}$ Exemple : une enquête nationale sur les Connaissances, Aptitudes et Pratiques (CAP) des exciseuses, effectuée à l'initiative du ministère de la Santé de juin à août 1999 et publiée en novembre 2001.
} 
d'une volonté du gouvernement djiboutien d'adhérer à des valeurs occidentalisées et, par conséquent, de la nécessité de se conformer aux exigences des instances internationales ${ }^{12}$. Pour autant, et les documents officiels ne manquent pas de le rappeler ${ }^{13}$, la loi interdisant ces pratiques n'a jamais été, jusqu'à présent, appliquée à Djibouti.

Abandonner la pratique des MGF participe à un bouleversement de l'organisation sociale djiboutienne. La loi, dans ce contexte particulier, est difficilement applicable et ne semble pas adaptée à la réalité socio-culturelle djiboutienne. En conséquence, les MGF sont encore largement pratiquées ce qui accentue encore la difficulté d'appliquer la loi :

"Dans chaque famille, il y en a qui s'adonne à cette pratique, qui perpétue cette pratique. Donc (...) si on commence à poursuivre, ça ne va plus s'arrêter (...) Le procureur, s'il poursuit quelqu'un, demain, ça peut être sa tante (...) C'est peut-être cette chaîne-là qui fait peur à tout le monde (...) Tous, ils nous ont blessé, fait du mal, mais en même temps, on est lié par le sang, par la chair. (...) En poursuivant quelqu'un, on poursuit tout le monde » (F4).

Il est peu concevable de condamner tout le monde et, avant même d'envisager une quelconque condamnation, il faut qu'il y ait eu, en amont, une dénonciation. Or, étant donné que seuls les proches de la victime ou la victime elle-même ont le droit de porter plainte contre la personne ayant pratiqué la mutilation, qui osera prendre la responsabilité d'effectuer la dénonciation ? Lorsque la victime atteint l'âge légal de porter plainte, c'est-à-dire dix-huit ans, les faits sont, dans la plupart des cas, trop anciens pour être dénoncés. Il y a donc prescription. En outre, si victime et coupable sont unis par les liens du sang, toute dénonciation revient à faire tomber le déshonneur sur leur propre famille. La conséquence ne serait pas uniquement individuelle - condamner un individu à une amende - elle serait sociale.

Dès lors, la loi, censée sanctionner la pratique des MGF d'une part et légitimer la volonté des autorités politiques de lutter contre celle-ci d'autre part, perd toute signification.

\section{- Une mobilisation collective insuffisamment visible dans l'espace public}

La lutte contre les MGF est réalisée grâce à la diffusion de messages dans les médias : émissions ou messages à la radio et à la télévision, articles dans la presse, séances de sensibilisation dans les quartiers, conférences. L'ensemble des personnes rencontrées au cours de notre recherche a témoigné de la dynamique de cette mobilisation. Cependant l'absence de messages écrits, d'affiches, d'illustrations, de panneaux d'informations sur la voie publique, sur les bâtiments des administrations et institutions, impliquées ou non dans la lutte mais représentant l'Etat, ou encore l'absence de distribution de prospectus, rendent cette mobilisation peu visible sur la scène publique. La lutte contre les MGF semble donc reléguée dans des espaces confinés : la sensibilisation a lieu dans des espaces-temps formels tels que des locaux associatifs, des salles de conférence, des centres de soins, etc. Autant de lieux prévus en partie à cet effet et fréquentés par une minorité de la population elle-même souvent

\footnotetext{
12 La Déclaration et le Programme d'action de Vienne, issue de la Conférence mondiale sur les droits de l'homme qui s'est tenue à Vienne en 1993, sous l'égide des Nations unies, appelle à l'élimination de toutes les formes de violence contre les femmes. Cet objectif est alors considéré comme une nécessité au regard de la défense des droits fondamentaux. En outre, la déclaration des droits de l'homme des Nations Unies et les nombreuses normes internationales qui en découlent mettent en évidence l'obligation qui est faite aux Etats de respecter et de faire respecter les droits fondamentaux de la personne humaine, comme le droit à l'intégrité physique et mentale, le droit de ne pas être l'objet de discrimination en fonction de son sexe, et le droit à la santé. Enfin, un certain nombre de normes internationales plus récentes, notamment des traités ratifiés par de nombreux pays, interdisent explicitement les MGF. La question est inscrite sur la liste de la sous-commission de la lutte contre les mesures discriminatoires et de la protection des minorités de l'ONU depuis le début des années 1980.

${ }^{13}$ PNUD, Rapport national sur le développement humain, 2004.

Ministère de la Promotion de la femme, DRAFT de la Stratégie Nationale pour l'abandon de l'excision et de l'infibulation, Djibouti, septembre 2006.
} 
déjà sensibilisée sur la question, voire conquise par la cause. La diffusion de messages à la radio ou à la télévision, quant à elle, permet sans doute l'introduction de la question des MGF dans l'espace privé. Cependant, libre à chacun d'éteindre son poste de télévision ou de radio pour ne pas entendre parler de ce sujet délicat.

En outre, à cette délimitation spatiale s'ajoute une délimitation temporelle puisque la sensibilisation est uniquement assurée par des personnes physiques - a contrario des supports papiers - donc à des heures précises, préalablement définies. Enfin, cette sensibilisation s'effectuant par voie orale, l'information peut être oubliée, déformée ou niée. Nulle trace écrite ne permet de pérenniser les messages diffusés.

Cette limitation spatio-temporelle révèle un décalage entre l'engagement des institutions et la difficulté d'aborder le sujet des MGF dans l'espace public même par les autorités politiques. La question des MGF dérange et est, par conséquent, difficile à introduire et à exposer de manière constante sur le devant de la scène publique. Cependant, ce manque de visibilité limite largement l'impact de la sensibilisation. En effet, comment faire réagir la population sur la question des MGF si celle-ci reste invisible, dans l'ombre du tabou et du respect de l'ordre social traditionnel?

\section{- Un tabou, frein à l'évolution des pratiques}

Aborder la question des MGF, c'est par définition aborder des thématiques liées au corps de la femme, au sexe, à la sexualité, au plaisir sexuel. Par conséquent, la lutte contre les MGF nécessite d'exposer et de rendre public ce qui relève à Djibouti de l'ordre privé, de la sphère du caché, de l'intime, voire de l'interdit. Il s'agit donc de parler et d'exposer en public, ce qui, au contraire, si on veut être respectueux de l'ordre, doit être tû, dissimulé, relégué hors de portée des regards. Il s'agit donc de parler de ce dont il convient de ne pas parler : le sexe et la sexualité, d'où la difficulté de traiter ouvertement et de manière claire et approfondie de la question des MGF.

Le tabou ne réside pas tant sur les MGF elles-mêmes que sur le sexe et la sexualité. Les femmes parlent facilement de MGF, du moins entre elles. En témoignent par exemple les réunions d'associations, leur présence aux conférences, leur volonté de participer à notre enquête, leur engagement associatif. En revanche, décrire la pratique des MGF elle-même, les différents types de mutilations, parler de sexualité et de plaisir sexuel, s'avère beaucoup plus délicat. Les femmes, au cours de nos entretiens, en parlent avec beaucoup de réserve. Au niveau du langage, la pudeur se manifeste comme un processus inhibiteur qui freine ou empêche toute formulation explicite ou désignation claire de l'objet auquel on veut faire référence. L'emploi récurrent d'euphémismes pour désigner le clitoris est révélateur : des termes tels que «le truc», "la chose» permettent de désigner sans choquer. Outre l'impossibilité à nommer ce qui constitue le cœur du tabou, les individus ont tendance lorsqu'on aborde ces sujets sensibles à se replier physiquement sur eux-mêmes. Le tabou fondamental est bien celui du sexe. Les MGF, quant à elles, parce qu'elles consistent en des interventions directes sur le sexe de la femme, deviennent tabou par contamination.

Par ailleurs, le tabou apparaît dans le souci constant des autorités d'éviter toute atteinte à la pudeur. Cette préoccupation limite là encore la lutte contre les MGF qui, par principe, n'est pas ouvertement menée sur la scène publique. Enfin, c'est parce que la question des mutilations génitales féminines pose en termes brutaux celle de l'aliénation de la femme dans les systèmes patriarcaux et donc, apparaît comme le paradigme de toutes les oppressions féminines qu'elle reste plus ou moins dans l'ombre.

La difficulté de briser le tabou traduit les contradictions internes du pouvoir qui d'un côté entend s'engager clairement dans la lutte contre les MGF, et de l'autre côté est réticent à 
heurter une partie du corps social en brisant le tabou. Enfin, elle pose également la question de l'intention réelle des institutions porteuses des projets de sensibilisation à aborder la question des MGF de manière frontale. Le souci de ménager la susceptibilité de certains acteurs sociaux (leaders religieux, hommes) historiquement porteurs de l'autorité, s'il évite les soubresauts sociaux, ralentit aussi la prise de conscience généralisée de la population.

\section{- Les MGF : une pluralité de définitions}

L'expression «MGF », qui recouvre l'ensemble des pratiques reconnues à Djibouti sous les noms d'infibulation ou "excision pharaonique », d'excision et de sunna, a été adoptée en raison de la gravité et du caractère irréversible des lésions qui sont infligées aux fillettes. Le consensus apparent que revêt cette définition ne doit pas masquer quelques divergences de sens qui ne manquent pas de susciter quelques confusions.

Les mutilations génitales féminines sont parfois désignées à Djibouti sous le terme de «circoncision féminine $»^{14}$. Cette appellation établit un parallèle direct avec la circoncision masculine qui prête à confusion entre deux pratiques pourtant bien distinctes. Elle tend à minimiser les risques des MGF. L'emploi du terme de circoncision est effectivement moins lourd de sens que celui de mutilation. Ce dernier est directement associé à la souffrance, à la violence. La circoncision, elle, est associée à l'idée de rite et figure comme une pratique sans risque aux yeux de tous, y compris la communauté internationale. En outre, souvent justifiée par des raisons d'hygiène, la circoncision masculine revêt une connotation positive. L'emploi du terme de "circoncision féminine », occultant la souffrance assignée aux fillettes, est, par conséquent, plus accepté et acceptable par les populations concernées qui ont du mal à accepter l'idée selon laquelle elles « mutilent» les fillettes.

Enfin, parler de «circoncision féminine » tend également, grâce à un glissement sémantique, à légitimer la pratique des MGF. Car la circoncision masculine ne fait pas, elle, l'objet de réprobation ou de condamnation. Elle est totalement légale. Par conséquent l'emploi du terme de «circoncision féminine» implique que les MGF sont une pratique acceptée et reconnue par tous. En définitive, l'inadéquation du terme «circoncision féminine » désignant les MGF introduit une ambiguïté quant à la signification même de ces pratiques.

Par ailleurs, les agences des Nations Unies et les institutions nationales pensent que l'expression de « Mutilations Génitales Féminines » ou le terme de " mutilation » véhicule un jugement négatif à l'égard des communautés qui les pratiquent. Par conséquent, elles ont adopté, depuis peu, une nouvelle terminologie.

L'UNFPA, pour sa part, soutient la définition des MGF de l'OMS ${ }^{15}$ qui recouvre sous le terme de Mutilations Génitales Féminines, tous types de MGF.

De son côté, l'UNICEF emploie désormais le sigle « E/MGF». Ce dernier signifie « Excision/ mutilation génitale féminine ». Une distinction est donc opérée entre excision et mutilation. Ce choix sémantique pose deux questions qui auront des conséquences sur la conception ultérieure des campagnes de sensibilisation : l'excision n'est-elle plus considérée comme une mutilation? Le terme de MGF ne recouvre-t-il donc que la pratique la plus sévère, c'est-à-dire l'infibulation?

Le Gouvernement djiboutien, quant à lui, dans la stratégie nationale de lutte contre les MGF, a remplacé l'expression de «MGF» dans ses textes par celui d' « excision et infibulation». Les MGF sont donc définies selon deux types de pratiques et non plus quatre. Cette nouvelle

\footnotetext{
${ }^{14}$ Rapport de l'enquête PAPFAM, 2002. Enquête « Fréquence, complication et stratégie d'intervention », 2003.

${ }^{15}$ La classification de l'OMS est la suivante : Type 1 : excision du prépuce, avec ou sans excision partielle ou totale du clitoris ; type 2 : excision du clitoris, avec excision partielle ou totale des petites lèvres ; type 3 : excision partielle ou totale des organes génitaux externes et suture/rétrécissement de l'orifice vaginal (infibulation); type 4 : piqûre, perforation ou incision du clitoris et/ou des petites et des grandes lèvres, étirement du clitoris et /ou des lèvres, cautérisation par brûlure du clitoris et du tissu avoisinant.
} 
terminologie semble exclure les formes de mutilations les plus légères. Dans ce sens, elle est problématique puisqu'elle semble implicitement impliquer l'idée que la sunna n'est plus considérée comme une MGF et donc, a fortiori, qu'elle est autorisée et socialement acceptable. L'ambiguïté reste à éclaircir.

Enfin, les témoignages recueillis au cours de notre enquête attestent de l'ambiguïté de la définition des MGF. Les individus confondent les diverses pratiques, certains doutent même de ce qui interdit, recommandé ou autorisé.

L'ambivalence des définitions du terme de «MGF» contribue à amoindrir la lisibilité des objectifs de la lutte contre les MGF et compromet l'efficacité des campagnes de sensibilisation contre cette pratique. Car, comment exiger un changement de pratiques si celles-ci ne sont pas ou mal définies et si les institutions porteuses des messages de sensibilisation ne s'accordent pas sur une définition univoque et, a fortiori, sur l'objet précis de la lutte?

\section{1-2 : Des contradictions internes}

\section{- Des pratiques néfastes réalisées en milieu hospitalier}

A la demande des familles, il arrive que les MGF, bien qu'illégales, soient pratiquées, par des praticiens du corps médical et parfois même en milieu hospitalier. Cette médicalisation des pratiques, bien que réalisée en vue de minimiser les risques de l'opération tend à légitimer la pratique des MGF puisque celle-ci est réalisée dans un espace public : 1'hôpital, entité du Ministère de la santé, administration de l'Etat (donc symbole de la politique et de l'action de celui-ci). La pratique des MGF en milieu hospitalier suggère donc que l'Etat tolère ce type d'intervention et, par conséquent tend à décrédibiliser lui-même sa volonté d'éradiquer ces pratiques.

Par ailleurs, rappelons que l'hôpital, en tant qu'institution, et que les médecins, en tant que praticiens, ont pour vocation - pour le moins dans les conceptions occidentales mais qui ont été transposées dans le contexte djiboutien - de soigner les individus et d'éviter la souffrance. Dans cette optique, ils sont tenus de divulguer et de mettre en auvre les messages d'information visant à la santé et au bien-être des populations. La pratique des MGF en milieu hospitalier est donc, par définition, un non-sens.

Cette médicalisation de la pratique des MGF révèle une incohérence entre la volonté politique affichée d'abandonner la pratique des MGF et ce qu'il se passe effectivement dans la réalité de l'espace étatique.

\section{- L'ambivalence des discours religieux}

La pratique des MGF est, dans l'esprit des Djiboutiens, largement associée à l'Islam puisqu'elle serait, selon eux, une recommandation du prophète Mahomet. La perpétuation de cette pratique repose ainsi sur des croyances religieuses. Cependant, officiellement, "la position des leaders religieux djiboutiens est clairement celle d'une condamnation de toute formes de $M G F »$ (PNUD, 2004; 67) puisqu'aucune base coranique n'en justifie la perpétuation.

Toutefois, cette position reste ambivalente dans la mesure où les leaders religieux estiment que la sunna est différente d'une mutilation génitale féminine. Leur condamnation n'équivaut donc pas à une condamnation de tous les types de pratiques. Cette condamnation partielle contrecarre le discours des politiques et, a fortiori, la sensibilisation des populations djiboutiennes à la question des mutilations génitales féminines. 
En outre, les discours des leaders religieux expriment des positionnements très variables sur cette question. Certains chefs religieux s'expriment en faveur de l'éradication de tous types de MGF tandis que d'autres attestent d'une éradication partielle puisqu'ils défendent et recommandent la pratique de la sunna au nom de l'Islam. L'hétérogénéité de ces discours suscite d'autant plus confusion dans l'esprit des Djiboutiens que la légitimité des Cheikhs est certaine et, par conséquent le poids de leur parole est non négligeable. En définitive, comme l'explique l'une de nos enquêtées, «Dans l'esprit de la population, la confusion persiste (...) Les gens ne sont pas convaincus que cette pratique soit interdite par la religion » $(\mathrm{F} 4)$. La divergence des discours des religieux sur la question des MGF constitue ainsi une entrave à l'évolution de ces pratiques.

\section{- L'emploi du terme de "sunna ", une ambigü̈té constante}

La forme la plus légère d'excision est appelée, à Djibouti, «sunna ». Or, littéralement, le terme de «sunna» signifie « recommandation». Cette terminologie renvoie directement au Coran. Ce qui est sunna pour les (Djiboutiens) musulmans, c'est ce qui est recommandé par la religion.

"Sunna, c'est un précepte, c'est un mot arabe. Il y a deux choses chez les musulmans : il $y$ a les trucs obligatoires et les recommandations. Donc les gens, ils disent, même qu'il n'y a pas vraiment de choses très claires, ils prétendent que la pratique de la sunna est une recommandation. Donc si cette pratique a été recommandée par notre religion, pourquoi on l'abandonnerait? ». (H9)

L'utilisation du terme de sunna pour désigner le type 3 de mutilation introduit est contradictoire avec l'idée selon laquelle les MGF ne répondent pas à une injonction religieuse. De surcroît, ce terme renvoie à l'idée de tradition, de comportement valorisé alors que l'on cherche justement à marquer une rupture avec ce comportement. Une telle contradiction émet là encore une ambiguïté source de confusion pour la population.

\section{- Une question de société, une sensibilisation axée sur les femmes}

Les MGF sont désormais considérées à Djibouti comme une question de société, face à laquelle tous, femmes et hommes, doivent prendre position. Pourtant, les discours et les messages de sensibilisation s'adressent uniquement aux femmes.

Dans la conception djiboutienne, les femmes sont, en tant que fillettes victimes de mutilations puis, mères de famille susceptibles de reproduire cette pratique sur leurs propres filles, les premières personnes concernées par la question des MGF. Nos interlocuteurs s'accordent à dire que l'homme n'a pas à charge la gestion de l'excision de ses filles et qu'il n'est pas concerné par la question.

Être concerné par les MGF semble se réduire alors au fait d'avoir subi dans sa chair la mutilation, et peut-être plus encore, au fait d'avoir perpétué la pratique, donc d'assumer un rôle social. Pourtant, peut-on affirmer que, parce que les hommes eux-mêmes, ne subissent pas de mutilations, ils ne sont pas concernés par la question?

Les hommes ne sont pas impliqués directement : certes, ils ne sont pas là physiquement le jour de l'opération. Ils ne participent ni à l'opération, ni à son organisation, ni aux soins postopératoires. Cependant, ce sont leurs filles, leur potentielle conjointe, ou leur femme qui sont les victimes. C'est la vie de leur proche, la sexualité et la fertilité de leur femme qui sont mises en péril. Par conséquent, en tant que père, mari ou futur époux des victimes - qui plus est : évoluant dans une société patriarcale, dominée par les hommes - ils sont concernés par 
cette question. Leur absence sur les lieux de l'opération ne signifie pas leur absence, en amont, dans le processus de décision d'exciser ou non leurs filles.

Enfin, cette pratique touche directement le domaine de la sexualité et donc les hommes en tant que partenaire sexuel. Parce que les femmes sont amputées d'une partie de leurs organes sexuels, leur plaisir sexuel est atténué. Un argument peu évoqué mais sous-jacent aux mutilations génitales féminines a toujours été le contrôle des pulsions sexuelles de la femme. Derrière la multiplicité des raisons ou des rationalisations pour justifier de cette pratique, prévaut le fantasme que la femme est un être dangereux pour l'homme et pour l'ordre masculin ${ }^{16}$. La croyance, souvent appuyée sur des mythes anciens, que la femme a une sexualité débordante et qu'il faut pour l'homme, sous peine de s'y perdre, arriver à la juguler, est encore vivace. Seule l'excision permettrait de rétablir l'équilibre en calmant les ardeurs des jeunes filles. Cette pratique limite (ou plutôt est supposée limiter car on ne voit pas comment, effectivement, l'excision empêcherait une relation extra conjugale et/ou un rapport sexuel) les relations extraconjugales, assure aux hommes la fidélité de leur femme et l'origine de leur progéniture. Enfin, elle conforte une garantie sociale (mais non biologique, génétique) sur la filiation. En définitive, l'angoisse de l'homme devant les incertitudes de la paternité, de la fidélité en miroir à son propre comportement puisque lui connaît des relations extraconjugales, la virginité de ses filles, la volonté de brider l'autonomie de la jouissance féminine si redoutable pour l'ordre patriarcal se dissimulent derrière ces pratiques.

En outre, avec l'infibulation, c'est le corps même de la femme qui devient une prison (nul, autre que son mari, n'a droit d'y pénétrer) que seul un homme peut ouvrir. Par conséquent, l'imposition de l'infibulation comme son déchirement relèvent d'une violence physique et psychique socialement légitimée, qui dépossèdent complètement la fillette et la femme de son corps et qui, en revanche, permettent à l'homme de mieux s'imposer. C'est pourquoi on peut voir dans la pratique des MGF l'expression de la domination masculine, ce qui introduit un lien social et culturel entre les hommes et la pratique des MGF.

\section{1-3: Un entre-deux générateur de tensions}

\section{- L'ambivalence de l'objectif initial}

Parmi les initiateurs de la lutte contre les MGF, certains aspirent à un changement radical des pratiques (exemple : ministère de la Promotion de la femme, UNFPA...) et d'autres à une évolution de celles-ci (exemple : l'UNICEF). Les premiers soutiennent l'éradication de tous types de MGF tandis que les seconds prônent l'abandon des formes les plus sévères d'excision, celle-ci constituant une étape transitoire avant même d'imposer l'interdiction de tous types de MGF. Deux logiques différentes sont à l'œuvre et renforcent l'ambiguïté. Les messages diffusés diffèrent ainsi selon la source dont ils proviennent.

Ainsi, deux types de discours ont été recueillis au cours de cette étude : l'un maintenant que la pratique de la sunna est autorisée, l'autre attestant de l'interdiction de tous les types de MGF. De cette confusion initiale découle des doutes et des interrogations quant aux pratiques réellement interdites. Il en résulte également une incompréhension et l'interprétation biaisée des messages diffusés ${ }^{17}$. Cette ambiguïé suscite l'adoption de comportements hétérogènes puisque les uns arrêtent la pratique de tous types de MGF, d'autres continuent de pratiquer la

\footnotetext{
${ }^{16}$ P. BOURDIEU, La domination masculine, Paris, Seuil, 1998.

17 L'ambiguïté réside dans le contenu des messages : certains prônant l'abandon de tous types de MGF, d'autres l'abolition de la seule infibulation. Cette ambiguïté est également celle des discours des chefs religieux précédemment.
} 
sunna. Une telle ambivalence laisse entrevoir un modèle fragile, peu structurant et, par conséquent, ni rassurant, ni attractif pour la population djiboutienne.

\section{- La non-application de la loi, une entrave à l'abandon des MGF}

La transgression de l'article 333 du code Pénal, bien que fréquente à Djibouti, n'est pas punie. En conséquence, elle devient la norme de comportement, c'est-à-dire le cas le plus usuel. Tout se passe comme si les individus n'avaient même pas à s'affranchir d'une loi pour perpétuer ces pratiques : ils ne sont pas considérés comme déviants ; un acte illégal est commis, mais il n'est pas perçu socialement comme une transgression des normes car le non-respect systématique de la Loi n'entraîne aucune réaction de la part de l'autorité chargée d'exercer le droit, pas plus que de réactions venant du corps social. En conséquence, les individus continuent de pratiquer les MGF sans éprouver de crainte de se voir punir. Cette nonapplication de la loi par l'Etat et l'absence d'une quelconque réaction dans l'opinion publique favorise la perpétuation des MGF.

En outre, parce que la loi interdisant les MGF n'a jamais été appliquée à ce jour ${ }^{18}$, elle constitue pour les Djiboutiens une abstraction théorique qui relève uniquement de discours. En revanche, la norme sociale ${ }^{19}$ a la force de la tradition et renvoie à des pratiques effectives, vécues et constatées depuis des générations. Elle constitue donc un cadre de référence plus solide pour la population que le nouveau modèle qu'est l'abandon des MGF. Ainsi, la norme sociale concurrence largement la loi juridique qui n'a jamais eu de légitimité aux yeux de la communauté. En définitive, parce que la loi juridique n'est pas, à ce jour, effective, elle n'est pas en mesure de s'imposer et de prendre le relais de la norme sociale qui, elle perdure.

Enfin, la non-application de la loi discrédite l'Etat aux yeux du «groupe pionnier» qui s'oppose aux MGF, car il n'assume pas ses fonctions, c'est-à-dire la mise en œuvre d'une loi qu'il a lui-même décidée. L'État, après avoir mis en place la loi interdisant les MGF, semble se désolidariser des acteurs de la lutte qui s'évertuent à faire disparaître ces pratiques.

Ce constat négatif pose une question de fond: comment changer des comportements profondément ancrés dans l'histoire des groupes ethniques composant la société djiboutienne, si cette volonté de changement elle-même n'est pas portée par le gouvernement et les institutions qui sont chargés d'appliquer la loi (justice, police) ? Le fait que la loi soit promulguée mais non appliquée est une incohérence dont la population a parfaitement conscience et dont jouent ceux qui sont favorables à la pratique des MGF. En conséquence, la résonance de ce nouveau modèle de comportements est largement affaiblie. Un tel modèle - l'abandon des MGF - ne peut servir de socle à la projection des individus dans un avenir maîtrisable. La légitimité du nouveau modèle n'apparait pas de manière forte et explicite. Ce modèle suscite le doute et incite peu les individus à s'y référer. Il reste donc très largement concurrencé par le modèle traditionnel : la perpétuation des MGF.

Ces considérations montrent combien la société djiboutienne est complexe, transcendée par des tensions et des contradictions entre des acteurs pluriels : les religieux, les hommes et les femmes, les représentants de l'Etat. La pression internationale relayée par les instances nationales place les Djiboutiens, et particulièrement les femmes, devant un dilemme : reproduire ou abandonner les MGF, alors qu'aucun moyen leur permettant de prendre cette décision ne leur sont donnés. Les individus sont ainsi mis en porte-à-faux vis-à-vis de leur héritage, de leur culture, puisqu'on leur exige un changement de pratique sans leur donner de solutions (application de la loi, renforcement du statut de la femme...). Le terrain initial de la

\footnotetext{
${ }^{18}$ Date de cette enquête novembre-décembre 2006.

${ }^{19}$ La norme sociale renvoie au comportement qu'il est implicitement convenu d'adopter dans la société djiboutienne en matière de MGF, comportement dicté par les aînés et en conséquence, comportement le plus usuel.
} 
lutte contre les MGF s'avère confus et peu solide et rend d'autant plus complexe la prise de décision des individus d'exciser ou non leur(s) fille(s). Dans ce contexte initial ambigu, comment et par qui la décision est-elle élaborée?

\section{2- L’analyse du processus décisionnel}

Notre travail, qui vise à analyser le processus décisionnel à l'œuvre dans la société djiboutienne, les familles, les couples, au sujet de l'excision des fillettes a permis d'identifier des facteurs favorables à l'abandon de l'excision. Cependant, cette étude disqualifie les tentatives de désignation de facteurs uniques et stables. Elle met en évidence des configurations complexes de facteurs, évolutives dans le temps.

Plus que des facteurs déterminants, il existe des facteurs favorisant l'abandon des MGF. Chacun d'eux, mis en évidence dans le présent article, pris individuellement, ne suffit pas à lui seul à faire abandonner la pratique des MGF. C'est la combinaison de plusieurs facteurs qui seule, rend possible un changement de pratiques. C'est leur alliance et leur interaction qui déterminent le comportement des individus. De ce point de vue, tous ont une importance, mais aucun n'est pourtant indispensable. La décision d'abandonner la pratique des MGF résulte donc d'un faisceau complexe de raisons multiples dont nous tâcherons de rendre compte.

\section{2-1: Une prise de décision relative au statut des individus}

La prise de décision d'exciser ou non les fillettes est soumise à diverses influences que nous tenterons d'identifier et dont l'effet varie selon l'origine. Femmes, hommes, aînés ou cadets, extérieurs ou non à la cellule familiale, tous n'ont pas le même poids dans la prise de décision.

\section{- Le poids des femmes et ses limites}

Parce qu'elles en sont à la fois les victimes et les coupables, les femmes sont les premières concernées, voire les seules, par la question des MGF. En effet, selon une assertion du sens commun véhiculée par les femmes comme par les hommes, les MGF seraient « une affaire de femmes ». La question de l'excision des fillettes au sein d'une famille revient ainsi essentiellement aux femmes. Un renversement du pouvoir de décision des hommes vers celui des femmes s'opère dans ce domaine particulier.

Par exemple, l'un des hommes rencontrés (3), au cours d'un entretien, reconnait ses propres limites dans le domaine des MGF face aux femmes. Il semble presque désarmé face à ce groupe. Devant la menace d'enlèvement de sa fille, il renonce à s'opposer. Il délègue ainsi implicitement l'affaire aux femmes et se retire du débat. Un tel retrait du débat face à des femmes de la part d'un homme dans une société patriarcale pose question quant à la volonté de l'homme de s'investir dans le débat.

Cette soi-disant « affaire de femmes » est ainsi pilotée, au sein des familles, par le groupe des femmes associant souvent grand-mères, tantes et mère de la victime. Cependant, nos interlocutrices soulignent également les limites de leur marge de manoeuvre.

F3, dont nous avons examiné l'opposition avec les femmes de sa famille, a bien précisé qu'elle n'avait pas bénéficié du soutien de son mari et ce manque de soutien s'est avéré, selon elle, déterminant. Il semble en effet que les femmes doivent toujours obtenir la «permission, l'aval » de leur mari pour faire exciser leurs fillettes, ce qui veut dire que les hommes ont soit donné explicitement la permission, soit qu'en raison de la tradition, la permission est perçue comme évidente, implicite. En tout état de cause, ce constat est contradictoire avec le fait de dire que les MGF sont une affaire de femmes. 
Le poids des femmes semble donc pouvoir être contré par celui des hommes, si ceux-ci décident explicitement et fermement de s'opposer. Ce constat renforce l'idée selon laquelle les hommes laissent la décision aux femmes non pas tant parce qu'ils sont soumis à une pression, que parce qu'ils fuient ainsi cette question sensible et dérangeante.

En définitive, l'influence masculine n'étant pas revendiquée, les femmes disposent du pouvoir de décision. Mais, dès que les hommes s'impliquent, la configuration n'est plus la même.

\section{- De l'absence des hommes à leur possible implication}

Erlich note que dans la littérature scientifique, "il n'est pratiquement jamais question de l'appréciation globale de ces pratiques par les hommes appartenant aux sociétés concernées » (Erlich, 1986 : 170). Couchard va dans le même sens en constatant que : "Une question assez rarement posée est la réaction du monde masculin devant les mutilations sexuelles» (Couchard, 2003: 115). Cette exclusion des hommes est le résultat d'un positionnement scientifique, politique et institutionnel des acteurs impliqués dans la lutte contre les MGF, qui ont repris, à leur compte, sans esprit critique, l'idée socialement construite selon laquelle les MGF sont « une affaire de femmes ». Il est pourtant surprenant de parler d'absence des hommes dans une société où ils détiennent l'autorité et le pouvoir.

Dans la mesure où les individus évoluent dans une société patriarcale dominée par les hommes, ces derniers ont tout le loisir d'intervenir dans la décision d'exciser ou non leurs filles. Les quelques résistances des femmes auxquels ils pourront être confrontés s'ils tentent de reprendre le pouvoir de décision dans ce domaine spécifique auront vite fait d'être atténuées, la domination masculine sous-jacente reprenant rapidement le dessus. Par conséquent, si l'homme décide de s'impliquer dans la décision, il reprendra le pouvoir et imposera sa volonté.

Par ailleurs, l'homme a d'autant plus de pouvoir qu'il a un statut de père mais aussi de chef de famille. Ce statut particulier (chef et père de famille) lui confère une légitimité qui, ellemême, lui permet de s'imposer dans sa propre famille. Les hommes, et particulièrement les chefs de famille, peuvent non seulement s'impliquer dans la question des MGF, mais surtout imposer un changement d'attitude. Ce constat est également et tout particulièrement vérifié pour les hommes qui sont les aînés de leur fratrie.

En effet, le statut de fils aîné confère à l'homme une légitimité qui lui permet de s'imposer face à sa propre mère.

Dans la mesure où le pouvoir de décision diffère selon le statut de la personne qui soutient l'idée d'abandonner la pratique des MGF, les hommes, en tant que père ou chef de famille, ont un rôle central à jouer dans l'abandon de cette soi-disant « affaire de femmes ».

\section{- Un glissement de la prise de décision des aînés vers le couple conjugal}

\section{La légitimité des aînés à l'épreuve des évolutions récentes}

Les grands-mères ont eu longtemps un rôle prépondérant dans l'organisation de la pratique d'une MGF sur leurs petites-filles. Non seulement elles participaient à l'opération ellesmêmes mais elles en étaient aussi souvent à l'initiative. Les grands-mères rencontrées ont souligné l'évolution de leur rôle.

Alors que, d'une manière générale, une grand-mère était largement impliquée dans la vie de ses fils et de ses belles-filles, vivait dans le même foyer qu'eux, et avait entre autres choses à charge l'organisation de l'excision de ses petites-filles, la grand-mère, dans les conditions actuelles de logement urbain, vit indépendamment de ses enfants si elle n'est pas veuve. Les 
grands-mères prennent moins part à la vie du foyer de leurs enfants et régissent moins l'organisation du foyer de leurs fils du fait de leur séparation physique.

Le passage de la famille élargie à la famille nucléaire instaure une distance physique entre les générations : parents et enfants vivant séparément des grands-parents. Cette distance physique se répercute : les grands-mères ne sont plus au cœur des foyers et sont contraintes de faire la part entre ce qui les concerne directement et ce qui concerne le foyer de leur enfant.

L'implication moindre des grands-mères dans les foyers de leurs enfants, et particulièrement de ceux de leurs fils, se lit également dans l'organisation de la pratique des MGF. Certaines y participent encore physiquement, certes, mais en s'imposant moins qu'autrefois, laissant le choix à leurs enfants de pratiquer ou non une MGF sur leurs fillettes.

- "Moi, je leur ai dit, mais elles font ce qu'elles veulent. Moi, mon rôle, c'est de conseiller: "Il faut faire au moins ça ». Si elles le refusent, c'est leur problème. Je n'insiste pas à prendre la gamine, non! Parce que s'il arrive quelque chose, je serais responsable, alors ce n'est pas la peine. Donc, je ne m'impose pas 》 (F9).

- «Je ne toucherais pas, je laisserais, c'est son problème. C'est sous sa responsabilité, je ne vais pas le forcer. » (F6)

En outre, les grands-mères se démarquent de la responsabilité des parents, suggérant ainsi qu'elle n'est pas la leur:

- "C'est lui le responsable de sa famille, moi, je ne vais pas le forcer et je n'irai pas exciser sans son consentement ses filles (...) Même si je suis capable de le faire, je ne vais pas le faire parce que, ce qui est raisonnable, c'est que les parents me donnent leur accord. Parce que les parents sont les premiers responsables des enfants » (F5).

Enfin, la parole des aînés et plus précisément des grands-mères semble avoir perdu du poids :

- «Chez nous, on ne te considère plus... On te dit « Salam Aleikoum », mais sans plus.

Personne ne t'écoute. On ne te donne même plus le droit à la parole. Ça y est » (F6)

- «Maintenant, bon, tout a évolué. Je crois que les grand-mères, leurs paroles n'ont pas de poids. Peut-être que dans certaines familles, elles s'imposent toujours, mais dans les familles évoluées, chez les jeunes qui ont fait des études et tout ça, c'est au placard» (F9).

Les grands-mères se retirent donc progressivement du devant de la scène familiale laissant la place à leurs enfants et petits-enfants. Une fois les traditions transmises, elles ne cherchent plus à les faire respecter, laissant les nouvelles générations évoluer. La décision d'exciser ou non la fillette glisse ainsi progressivement des grands-mères vers le couple père/mère.

\section{Vers une prise de décision conjugale}

Coeur de la cellule familiale, le couple père/mère est de plus en plus reconnu libre et autonome par rapport au groupe. Ainsi, de la diminution de l'influence des grands-mères à l'émergence de la notion de couple, la cellule de décision, vis-à-vis des MGF tend à changer de configuration : le couple conjugal semble en être ou en devenir le centre.

Cependant, les femmes, plus que de parler de décision commune ont mis l'accent sur le fait que, dans un premier temps, elles décidaient de toucher ou non leur fille et que leur mari les suivaient dans leur choix. Ceci ne veut pas dire que la décision a été prise avec le père, qu'elle a suscité débat, mais simplement que le père a été informé avant de prendre une décision ferme et qu'il a validé le choix de sa femme.

Il semble donc que la décision soit, sinon négociée, au moins abordée au sein du couple conjugal. Ce déplacement du pouvoir de décision s'amorce à Djibouti. Il est signe que les hommes (des jeunes générations) sont concertés et que la pratique des MGF ou son abandon 
ne se fait plus à leur insu, comme c'était le cas pour la génération des actuelles grand-mères et mères de famille. La question d'exciser ou non les fillettes a donc été introduite dans l'espace privé au sein du couple conjugal. L'instauration d'un dialogue sur la question des MGF au sein du couple montre que cette pratique n'est plus une obligation, une norme à respecter mais une tradition à l'épreuve du changement. Cette évolution est en partie due à la mobilisation des associations sur cette question.

\section{- Les associations : leur rôle et leurs limites}

Parmi nos interlocutrices, certaines sont de près ou de loin, engagées dans la lutte contre les MGF à travers leur activité professionnelle (travail au sein de l'un des Ministères mobilisés) ou leur action associative. Leur investissement dans ce domaine se justifie par leur adhésion au nouveau modèle de référence ou, pour le moins, au refus de la perpétuation des MGF. Cependant, cette mobilisation ne se traduit pas systématiquement par l'abandon de la pratique au sein de leur propre famille.

Les femmes montrent, par un écart entre leur discours et leur pratique, qu'elles ne sont pas foncièrement convaincues par la cause qu'elles défendent ou, pour le moins, qu'elles ne parviennent pas à faire valoir des idées qu'elles défendent publiquement dans le cadre associatif ou professionnel, dans leur cellule familiale. On constate effectivement une rupture entre ce qui est dit, ce qui est prônée hors de l'espace domestique, et ce qui se décide dans la cellule familiale.

Ce constat interroge directement les convictions des acteurs associatifs et par-là même la crédibilité de leurs discours et de leurs actions. Des opinions défendues dans un lieu mais pas dans un autre sont des opinions peu consistantes ou, pour le moins pas assez, pour faire face à l'opposition, autrement dit des opinions mal assurées.

Enfin, la pression de l'extérieur, qu'elle vienne du voisinage, des associations, du cadre juridique, apparaît secondaire par rapport à la pression familiale.

\section{2-2 : Une décision déterminée par des facteurs individuels}

La décision d'exciser ou non les fillettes est empreinte d'éléments directement liés au contexte de vie des individus. Des « effets situationnels » précis orientent ainsi cette décision.

\section{- La réussite sociale de l'homme ou le pouvoir de décider}

Haut niveau d'instruction, insertion dans le milieu professionnel, fondation d'un foyer, construction d'une maison, achat d'une voiture sont autant de preuves d'une réussite sociale (c'est-à-dire sociale et économique). L'un de nos enquêtés, après avoir fait de longues études et vécu en France plus de cinq ans, occupe désormais un poste de cadre à Djibouti. Cette réussite sociale lui permet de bénéficier d'une certaine marge de manœuvre par rapport aux souhaits des membres de sa famille. Elle lui confère une autonomie (possibilité d'avoir sa propre maison), une indépendance (il devient la référence de la famille) et un pouvoir certain : pouvoir lié à la connaissance, pouvoir matériel (le reste de la famille en devient dépendant) et enfin un pouvoir de décision. Il acquière ainsi une certaine légitimité :

- «La réussite sociale donne une légitimité à n'importe qui! Au niveau de la famille, même du côté de mon père, par rapport à mon frère, j'ai une légitimité, je veux dire, vraiment une très grande légitimité » (H4).

Cependant, la réussite sociale n'est pas suffisante à conférer une légitimité. Cela dépend également du genre : la réussite sociale d'une femme, certes reconnue, l'est beaucoup moins que celle d'un homme. 
- «A Djibouti, on dira: "C'est avant tout une femme ». La société djiboutienne... Des femmes qui sont à des postes [hautement qualifiés], ce n'est pas pour autant que les hommes les considèrent, qu'elles ont une légitimité (...) Une femme qui a réussi socialement pourra imposer ses idées, mais encore faut-il qu'en face, il y ait des hommes qui comprennent et qui acceptent, ce qui n'est pas forcément le cas à Djibouti. Les hommes sont très machistes dans cette société. La femme peut réussir mais c'est une femme » (H4).

La discrimination à l'encontre des femmes à laquelle on peut conclure à la lecture de ce témoignage, est associée à une diminution de l'éventuel pouvoir dont les femmes pourraient bénéficier du fait de leur réussite sociale. Alors que la réussite sociale est synonyme de pouvoir pour les hommes, elle est sans doute plus limitée au simple respect pour les femmes. Enfin, les hommes bénéficient là encore d'un atout supplémentaire pour faire valoir leurs opinions au sein de leur famille.

\section{- Du bouleversement du système de référence au changement de pratiques}

La situation d'immigration engendre un bouleversement des normes de comportements et des systèmes de référence des individus. Ceci est d'autant plus vrai lorsque l'émigration se fait dans un pays occidental, qui plus est : en France, pays anciennement colonisateur de Djibouti et entretenant des liens étroits avec cette ancienne colonie, où la loi interdisant les MGF est appliquée (des condamnations ont déjà eu lieu), où la séparation de l'Etat et des religions fait partie de la Constitution, où hommes et femmes entretiennent des rapports d'égal à égal.

En effet, les migrants sont immergés dans une autre culture et évoluent au contact de populations qui ne fonctionnent pas de la même façon qu'eux, qui ont leurs propres codes de conduites et leur propre système de pensée. Toute migration constitue ainsi une situation d'interaction avec l'Autre, l'étranger, le différent. Au contact de cet Autre, le migrant est amené à revoir ses propres façons de faire, de dire, de penser de façon à s'adapter au mieux à sa société d'accueil. Dès lors, une question aussi singulière que celle des MGF est, en situation d'immigration, appréhendée différemment.

Par exemple, pour l'un de nos enquêté (H4), dont les enfants sont nés en France, la question de l'excision ne s'est pas posée avec la même acuité dans ce contexte d'immigration qu'elle l'aurait été s'il avait vécu à Djibouti durant l'enfance de ses filles.

A Djibouti, H4 aurait subi une pression sociale venant de sa famille, tandis qu'en France, la pression est avant tout d'ordre juridique. Les MGF y sont perçus comme une barbarie par l'opinion publique et des associations de femmes militent activement contre cette pratique.

Dans ce contexte, H4 s'est fié au comportement en vigueur dans son pays d'accueil. Bien qu'il ne s'oppose pas foncièrement à la pratique de la sunna, il a laissé ses filles telles qu'elles. Il n'a pas pris le risque de transgresser la loi française - largement appliquée - mais celui de contrecarrer la volonté de sa mère. Le risque de provoquer le conflit familial et de recevoir l'opprobre publique étant minime en France compte tenu de l'éloignement physique du migrant, il est dans l'intérêt d'H4 de s'en référer à la norme juridique et sociale de son pays d'accueil plus qu'à la norme sociale de son pays d'origine.

Dans la mesure où l'individu opère le choix de pratiquer ou non une MGF sur ses filles par rapport aux normes de référence en vigueur dans son pays d'accueil, le système de référence local apparaît déterminant. Un changement d'attitude passe par un changement de système de référence d'une part et une application ferme de la loi d'autre part.

Par ailleurs, la question des MGF, si elle ne se pose pas directement aux migrants dans leur société d'accueil (parce que leurs enfants n'y vivent pas), se posera lorsqu'ils seront de retour dans leur pays d'origine. Or, parce qu'ils ont connu une situation d'immigration, ces individus ont été amenés à réviser leur modèle de penser et d'agir intériorisé dans leur enfance. La 
confrontation avec l'étranger a en effet été l'occasion pour certains de nos enquêtés de se forger un regard réflexif sur leurs propres origines et un regard critique sur leurs propres façons de faire et de penser.

Ainsi, le contact avec les Français a permis à l'un de nos interlocuteurs (H3) de relativiser les idées socialement construites qu'il a intériorisées dans son pays d'origine. C'est également en France que $\mathrm{H} 3$ a pris conscience de la spécificité culturelle qu'est la pratique des MGF :

"J'ai découvert que ça ne se passait pas comme ça dans toutes les sociétés, dans toutes les cultures et qu'il y avait des cultures qui étaient étonnées de nous voir faire ce genre de choses, alors que pour nous, c'était normal (...). J'ai pris conscience de l'anormalité de ces pratiques en France et que j'ai changé d'opinion en France » (H3.)

Force est donc de constater que le contact continu et direct entre les Djiboutiens et leur société d'accueil entraîne des changements dans les modèles culturels initiaux des immigrés et favorise les changements d'opinions, voire d'attitudes. Cette acculturation aboutit à une réinterprétation des schèmes de pensées qui connaissent alors de nouvelles formes et de nouvelles significations. La migration, par l'acculturation qu'elle permet, participe à une restructuration des modes de pensées et d'agir des migrants. Ceux-ci, porteurs d'une nouvelle culture, de retour dans leur pays d'origine, contribuent à faire évoluer les pratiques qui y ont cours. La migration génère ainsi une réelle dynamique de changement et peut favoriser l'abandon de la pratique des MGF. Cependant, les migrants sont souvent des étudiants et des cadres, par conséquent, la migration renforce la conviction des personnes qui sont susceptibles d'être déjà convaincus en raison de leur niveau d'éducation.

\section{- La situation personnelle : un terrain plus ou moins propice au changement de pratique}

\section{La scolarisation}

L'enquête PAPFAM montre que l'attitude des femmes par rapport aux MGF est directement liée à leur niveau d'instruction, ce que les discours des enquêtés confirment. Il semble en effet que les individus ayant été scolarisés et ayant atteint le niveau secondaire disposent d'un terrain « intellectuel » plus propice à l'abandon des MGF qu'un individu non scolarisé (ou n'ayant connu que l'école coranique).

Les individus ayant atteint le niveau secondaire, parce qu'ils ont intériorisé une pluralité de normes, été instruits dans différents domaines de connaissance, sont plus à même de comprendre et d'intégrer les changements qui ont cours dans leur société ou leur groupe d'appartenance que les individus qui n'ont bénéficié d'aucun enseignement scolaire. L'introduction d'un nouveau modèle de référence dans une société est moins perturbante pour des individus qui ont déjà été confrontés à d'autres mondes de références, à l'altérité, parfois même à l'étranger, que pour des individus ayant été socialisés uniquement dans leur famille et, éventuellement, à l'école coranique.

En outre, les individus instruits, parce qu'ils ont accès au savoir : la connaissance du corps humain, de l'anatomie, de l'histoire, de la philosophie, ou des connaissances juridiques, sont plus à même d'appréhender les risques et parfois même les enjeux des MGF, ce qui favorise la remise en cause de ces pratiques.

Nous avons effectivement constaté que parmi les personnes interrogées dans le cadre de cette étude, des arguments tels que "l'intégrité physique et morale de la fille», ou le "risque de couper une veine lors de l'opération des fillettes " étaient évoqués par les personnes ayant bénéficié d'un enseignement secondaire. 
Enfin, on peut supposer que la capacité de réflexivité des individus instruits, à la différence des individus non scolarisés, leur permet de se distancier des préjugés et des messages parfois biaisés diffusés sur la scène publique, d'opérer un travail d'épuration et de clarification des données transmises et ainsi, de se forger une opinion ferme. A l'inverse, les individus ne disposant pas des outils intellectuels nécessaires à l'objectivation du monde dans lequel ils vivent, soit résistent à l'idée de bouleverser leurs acquis et leur monde de référence, soit s'imprègnent des préjugés, des rumeurs, des discours diffusés sur la scène publique au risque de se perdre dans le foisonnement d'informations et de mal interpréter les messages. Dans ces cas, ils adoptent des comportements conformistes. Or, le conformisme, par la rigidité qu'il suscite, ne facilite ni l'adaptation, ni la prévention du changement.

L'ensemble de ces considérations montre qu'un niveau d'instruction élevé favorise donc l'abandon de la pratique des MGF. Cependant ce seul niveau d'instruction est loin de déterminer à lui seul le comportement des individus en matière de MGF. Et inversement, une personne peut abandonner la pratique des MGF sans pour autant être instruite. Enfin, l'instruction n'exclut pas non plus la perpétuation des pratiques. Ce facteur n'est pas déterminant, ni suffisant, ni forcément nécessaire à l'abandon des pratiques, mais il le favorise.

\section{La socialisation}

Les témoignages de nos enquêtés montrent que le processus de socialisation ${ }^{20}$ selon qu'il est élaboré dans un environnement «traditionnel » ou plutôt " libéral » ${ }^{21}$, constitue un terrain plus ou moins propice au changement: changement de mentalité, changement d'attitude, changement de comportement.

Certains de nos interlocuteurs ont grandi dans une famille que l'on pourrait qualifier de conservatrice puisque très attachée aux valeurs traditionnelles. Ils n'ont connu que les bancs de l'école coranique. La division sexuelle des tâches est très marquée au sein même de la famille : les filles sont éduquées en vue d'être de bonnes mères et de bonnes épouses. Leur reconnaissance sociale passe par le mariage et la procréation. Les hommes quant à eux, sont éduqués dans les valeurs d'honneur et de courage, et chargés d'entretenir leur foyer. Ils détiennent le pouvoir et l'autorité. La conscience collective est très forte et prime sur l'individuelle. L'individu socialisé dans ce contexte montrera des résistances au bouleversement de ses cadres de référence.

D'autres ont grandi dans une famille plus souple et plus ouverte. Ils ont été scolarisés, voire ont prolongé leurs études au niveau supérieur. Ils ont été, au même titre que leurs parents parfois, au contact d'autres cultures lors de migrations temporaires. La personnalité de l'individu étant forgée au gré des contacts avec l'extérieur, l'introduction d'un changement, d'un nouveau modèle de référence s'opère progressivement.

\footnotetext{
${ }^{20}$ La socialisation désigne les processus par lesquels les individus s'approprient les normes, valeurs et rôles qui régissent le fonctionnement de la société. L'individu s'intègre ainsi dans une société donnée ou à un groupe particulier par l'intériorisation des modes de pensée, de sentir et d'agir qui y sont en vigueur. La socialisation dite primaire correspond à la période de l'enfance. Au cours de cette période, trois instances de socialisation vont contribuer à structurer la personnalité sociale du futur adulte : la famille, l'école et les groupes de pairs. La socialisation dite "secondaire " consiste aux processus postérieurs qui permettent d'incorporer un individu déjà socialisé dans des nouveaux secteurs du monde objectif de la société. La socialisation professionnelle en fait partie.

${ }^{21}$ Notons que qualifier les environnements des individus de «traditionnel » ou de «libéral » relève d'une catégorisation élaborée en vue de fournir un instrument d'intelligibilité du réel afin d'organiser, de clarifier, de mettre de l'ordre dans la complexité du réel. Par conséquent, la catégorisation suggérée représente deux types idéaux d'environnements qui représentent un cas limite, que l'on ne rencontre jamais dans sa pureté, mais à l'aune duquel on peut comparer les environnements réels qui s'en approcheront toujours plus ou moins.
} 
La remise en cause de la pratique des MGF suscite moins de résistance chez un individu socialisé dans un environnement «libéral» que chez un individu socialisé dans un environnement traditionnel, rigide.

\section{Les convictions, un générateur de changement de pratique?}

Se positionner pour ou contre une cause, c'est prendre le risque de s'opposer à autrui, donc de créer le conflit et briser l'unité de l'opinion. Compte tenu du risque encouru - d'autant plus accru dans la société djiboutienne puisqu'il s'agit d'une société fondée sur une certaine conformité, vecteur de cohésion sociale - si les individus s'engagent à prendre parti pour l'un ou l'autre modèle de comportement, c'est sans doute qu'ils sont convaincus de leur position et qu'ils sont prêts à la défendre malgré les oppositions et les résistances auxquelles ils feront face.

Notre étude montre en effet que les individus qui ont refusé d'exciser leur fille et pour lesquelles la question d'exciser ne se pose plus puisque leurs filles ne sont plus en âge d'être touchées (elles sont âgées de plus de douze ans) ${ }^{22}$, autrement dit les «pionniers », sont convaincus, à la différence de ceux qui affirment être contre ces pratiques mais qui les laissent se perpétuer au sein même de leur famille. Les pionniers agissent en fonction d'opinions fermes et durables.

Les convictions des individus sont repérables dans leurs discours et dans leurs actes. Les personnes concernées extraient la pratique des MGF de toutes significations symboliques et religieuses. La pratique est associée à une réelle " charcuterie » et n'apparaît plus qu'en tant qu'injustice et souffrance infligée à des innocentes. La consistance des opinions se lit essentiellement dans la capacité de résistance des individus à faire face à l'entourage en opposition à l'abandon des MGF. Enfin, parce que ces individus se disent « en paix avec [leur] conscience », "soulagés » d'avoir su imposer leur décision, on peut supposer qu'exciser leur fille aurait été à l'origine d'un conflit intra-individuel, source de tensions et de remise en cause difficiles.

Les seules convictions ne sauraient cependant suffire à elles seules à abandonner des MGF. Nous avons constaté que les femmes pionnières rencontrées avaient toutes le soutien de leur mari dans leur choix de ne pas toucher leurs filles. C'est donc la combinaison des convictions personnelles et du soutien du conjoint qui rend possible l'abandon des MGF.

\section{2-3 : De la pluralité des normes de référence à une prise de décision rationnelle}

Dans un contexte caractérisé par l'ambivalence, les individus ne parviennent pas à se positionner franchement pour ou contre les MGF. En effet, le contexte sociétal marqué par l'hétérogénéité des références sur la question des MGF ne facilite pas une structuration ferme et durable des opinions.

Les hommes, pour la plupart se retirent du débat. En adoptant des comportements de fuite, ils ne se prononcent pas sur la question, et ainsi, laissent se perpétuer les MGF. Des individus, parmi lesquels une large majorité de femmes, tentent de prendre position en s'engageant par exemple dans le secteur associatif, tandis que la majorité des personnes rencontrées adaptent leur positionnement au gré des circonstances, en fonction des normes auxquelles ils sont soumis.

\footnotetext{
${ }^{22} \mathrm{Ce}$ critère exclut donc les deux femmes que nous avons rencontrées qui disent avoir abandonné la pratique des MGF, dont les filles ne sont effectivement pas excisées mais qui sont âgées de six ans, donc susceptibles d'être excisées par la suite malgré les dires des parents.
} 


\section{- Une cellule de décision au carrefour de normes contradictoires}

A l'heure actuelle à Djibouti, les individus (pères, mères, grand-mère...) chargé de prendre la décision d'exciser ou non leur fillette sont au carrefour d'un ensemble de normes. Or, la société djiboutienne n'est pas une organisation homogène, où la définition des normes et leurs modes d'application dans des situations spécifiques feraient l'objet d'un accord unanime. C'est une société composite et multi ethnique. Chaque groupe ethnique a son propre système de normes. De plus, le changement social (la sédentarisation progressive des populations nomades, les contacts avec des sociétés occidentales, arabes et orientales, la généralisation de la scolarisation, les études supérieurs à l'étranger) et la différenciation économique de plus en plus marquée, introduisent de nouvelles références qui dépassent les spécificités culturelles ethniques. La société djiboutienne se caractérise par la superposition d'anciennes stratifications et de nouvelles hiérarchies sociales dont l'organisation ne se réfère pas aux mêmes systèmes de valeurs et ne relèvent pas des mêmes sources de légitimité.

La norme juridique nouvellement institutionnalisée concernant les MGF, loin d'être la seule référence pour les individus, entre en concurrence avec d'autres normes : religieuses, sociales, morales, familiales. Les témoignages des nos enquêtés révèlent la diversité des contraintes auxquelles ils sont soumis lors de la prise de décision. L'accent a été mis par nos interlocuteurs sur les contraintes sociales et religieuses d'une part et les croyances et représentations ayant trait à cette pratique d'autre part.

En outre, les individus au cours de leur trajectoire de vie sont parfois confrontés à différents mondes de références. Ils pensent être, du fait des mariages mixtes interethniques, qui ne se limitent pas à des personnes originaires de Djibouti (Ethiopie, Yémen, Somalie), les héritiers et les porteurs de plusieurs cultures qui ont des convergences et des divergences à la fois au niveau du système de valeurs et au niveau des pratiques (par rapport par exemple au statut de la femme, à la valeur de l'honneur, à l'importance du mariage). L'individu par définition est le produit de deux lignées, donc deux traditions familiales. Par conséquent, il est d'emblée cœur de plusieurs systèmes de normes qui peuvent aussi bien se combiner que s'opposer.

Comment donc, dans ce contexte, les Djiboutiens parviennent-ils à tenir compte simultanément de ces normes contradictoires ?

\section{- Une prise de décision rationnelle}

Tout individu chargé de prendre une décision est amené à opérer des choix, de manière plus ou moins consciente, plus ou moins intuitive, réfléchie ou spontanée, selon les contraintes qu'il rencontre. Les personnes interrogées adoptent certaines lignes de conduite selon leurs intérêts et le gain social qu'elles y trouvent. La décision de pratiquer une MGF sur une fillette semble ainsi s'inscrire dans une logique rationnelle.

Lorsque les individus choisissent de renoncer à pratiquer une MGF en invoquant comme principe premier la santé des fillettes, ils remettent en cause la cohésion de leur groupe d'appartenance. En effet, privilégier la santé des fillettes et des femmes, c'est-à-dire abandonner la pratique des MGF, constitue une prise de risques pour les individus : celle d'introduire au sein de leur famille, de leur ménage, de leurs lignées, voire de leur communauté et de leur quartier, le désordre, l'instabilité et le conflit. Effectivement, dans la mesure où une telle prise de décision s'exerce rarement en catimini, décider d'abandonner cette pratique ancestrale implique une négociation avec les aînés.

Par exemple, l'un de nos enquêtés dit lui-même avoir cédé face à sa belle-mère de peur de provoquer le désordre dans la famille. Pour lui, les conséquences de l'abandon des MGF sont plus lourdes que celles consécutives à la pratique. Même s'il est sensible au nouveau code de conduite exigé, étant donné que celui-ci n'est pas encore appliqué, cet homme ne prend pas 
le risque de transgresser la norme sociale. Bien que conscients des méfaits de ces pratiques, il a jugé nécessaire de s'y conformer au nom de la cohésion familiale et du respect de ses aînés.

Dans la mesure où la loi qui devrait contraindre les personnes à adopter le nouveau modèle de comportement n'est pas appliquée, les individus, en la transgressant ne prennent aucun risque. Ces individus ont donc plus intérêt à transgresser cette loi, puisque cette transgression est sans conséquence, qu'à transgresser les valeurs traditionnelles puisque cette transgression leur sera reprochée et les conséquences familiales et sociales seront importantes.

Le choix d'exciser ou non les fillettes est fonction de ce que chacun juge prioritaire au moment de prendre la décision. C'est ainsi que, rationnellement en fonction du système de contraintes dans lequel ils vivent, des individus perpétuent la pratique des MGF. L'exemple qui suit est éloquent.

En effet, lorsque F3 s'est vue confrontée à l'excision de sa propre fille, elle s'est foncièrement opposée aux autres membres de sa famille (mère, sœurs, tantes, conjoint...) afin de les dissuader de «torturer» sa fille. Une phase de négociation a donc été amorcée entre F3 et sa mère. Discussion, pleurs, éloignement de F3 des siens, n'ont pas suffit à cette femme pour faire valoir ses idées et épargner sa fille. Car, la négociation s'est achevée lorsque sa propre mère en est venue aux menaces. C'est à la menace, au chantage, de sa mère : "on va lui faire un truc pire encore » que F3 n'a pu résister. Elle a cédé optant pour la situation « la moins pire ». Le choix contraint de F3 résulte d'une évaluation des risques pour chaque possibilité : faire valoir ses idées, privilégier l'intégrité physique de sa fille au risque que celle-ci subisse ensuite une mutilation sévère faite de force par les grands-mères ou, se résigner, céder à la volonté de sa mère, mais faire subir à sa fille une forme de mutilation plus légère moins risquée que celle dont on la menace. F3 choisit donc la situation de laquelle elle tire le moins d'inconvénients : la pratique de l'excision.

La prise de décision d'exciser ou non les fillettes résulte donc d'une évaluation des contraintes qui pèsent sur l'individu à un moment donné, des possibilités de soutien dont l'individu peut ou non bénéficier dans sa famille ou par les réseaux associatifs. Les individus adoptent avant tout un comportement en fonction des diverses contraintes qui pèsent sur eux.

L'abandon de la pratique des MGF résulte d'une décision collective impliquant les femmes de la famille et en particulier la mère de la fillette, à laquelle est associée le consentement d'un homme mais pas systématiquement celui d'une femme, ni même celui d'un(e) aîné(e). Autant la perpétuation de la pratique peut se faire à l'insu de l'homme, autant l'abandon nécessite son accord dans la mesure où l'homme constitue un soutien indispensable à une mère face à son entourage. La décision d'une femme est ainsi légitimée par le soutien de l'homme. L'homme peut, quant à lui, décider seul d'abandonner la pratique des MGF. Son statut d'homme dans une société patriarcale lui permet effectivement d'asseoir ses décisions, de manière autonome et à son gré.

La décision de pratiquer ou non une MGF, pour la plupart de nos enquêtés, ne relève pas tant de convictions que d'éléments issus d'un contexte particulier. L'imposition d'une problématique aussi délicate et lourde à gérer que celle de l'abandon des pratiques des MGF, à des individus très inégalement préparés à la maîtriser, suscite en réalité des réponses qui résultent d'un ensemble de divers critères. La décision résulte d'un compromis entre le contexte initial ambigu, les normes contradictoires auxquelles les individus sont soumis, et des facteurs individuels. Cette décision, empreinte de contexte de vie plus que d'opinions, ne permet pas à tous les individus d'adopter des comportements conformes à leurs discours. Tous les individus ne sont pas placés dans un contexte familial, socio-culturel et économique leur permettant de mobiliser les ressources et les alliés nécessaires à faire prévaloir leur position anti-MGF. Ces comportements sont en définitive un ajustement à un contexte initial 
ambigu. Car, à ce contexte initial ambigu, les individus répondent par l'ambiguïté en pratiquant la sunna, une pratique intermédiaire dans la mesure où les fillettes sont soumises à une intervention mais où leur souffrance est moindre. Cette pratique apparaît ainsi comme un compromis entre les deux modèles de référence.

\section{$\underline{\text { Bibliographie }}$}

\section{Ouvrages}

BOURDIEU P., La domination masculine, Paris, Seuil, 1998.

COUCHARD F., Le Fantasme de séduction dans la culture musulmane. Mythes et représentations sociales, Paris, PUF, 1994.

COUCHARD F., L'excision, Paris, PUF, Que sais-je, 2003.

ERLICH M., La femme blessée : essai sur les mutilations sexuelles, Paris, L'Harmattan, 1986.

PROLONGEAU H., Victoire sur l'excision. Pierre Foldes, le chirurgien qui redonne l'espoir aux femmes mutilées, Albin Michel, Paris, 2006.

\section{Enquêtes et rapports}

Direction de la Statistique et des études démographiques (DISED), « Enquête djiboutienne sur la santé de la famille, PAPFAM », Rapport principal, 2004.

Direction de la Statistique et des études démographiques (DISED, «Fréquence, complication et stratégie d'intervention », Rapport d'enquête, 2003.

Ministère de la Santé, «Enquête nationale sur les Connaissances, Aptitudes et Pratiques (CAP) des exciseuses », 2001.

Ministère de la santé, "La fréquence, les complications et les stratégies d'intervention », 2002.

Ministère de la santé, « Stratégie Nationale pour l'abandon de l'excision et de l'infibulation », DRA FT réalisé en collaboration avec l'UNICEF, septembre 2006.

PNUD, Rapport national sur le développement humain, 2004.

POPINTER, UNFPA, La médecine scolaire à Djibouti, Enquête pilote 2005-2006, Paris, 2006, 24 p. 